\title{
Autoimmune thyroid disease with ulcerative colitis
}

\author{
Onyechi Modebe
}

Department of Medicine, White Memorial Hospital, Los Angeles, California, USA.

\begin{abstract}
Summary: Two cases of co-existing thyroid disease and ulcerative colitis are reported. Thyroid disorder preceded ulcerative colitis in each case. The presence of acute colitis delayed and obscured the clinical diagnosis of thyrotoxicosis in one case and the colitis could not be controlled until her thyrotoxicosis was treated. Although the specific factors involved in this relationship are now known, an interplay of immunological factors is most probable.
\end{abstract}

\section{Introduction}

The association of Graves' disease and ulcerative colitis has been reported infrequently. Edwards \& Truelove (1964) and Janerot et al. (1975) have noted a significantly increased frequency of thyrotoxicosis in patients with ulcerative colitis and Powell et al. (1968) have emphasized the difficulty of treating ulcerative colitis in the presence of hyperthyroidism. Two patients are reported who developed ulcerative colitis while being followed for thyrotoxicosis; one of them had an exacerbation of her ulcerative colitis with the recurrence of the thyrotoxicosis.

\section{Case reports}

Case 1

A 41 year old black woman complained of a neck mass and recurrent episodes of periorbital swelling and leg cramps for 7 years. Physical examination showed proptosis, chemosis, ophthalmoplegia and a diffusely enlarged, firm, non-tender goitre.

Her haemoglobin was $11.5 \mathrm{~g} / \mathrm{dl}$. Radioactive iodine uptake was $8.5 \%$ at 2 hours and $33.1 \%$ at 24 hours. Serum triiodothyronine (T3) was $150 \mathrm{ng} / \mathrm{dl}$ (normal range $80-160 \mathrm{ng} / \mathrm{dl}$ ), thyroxine (T4) was $8.0 \mu \mathrm{g} / \mathrm{dl}$ (normal range $5-12 \mu \mathrm{g} / \mathrm{dl}$ ), and $\mathrm{T} 3$ resin uptake was $27.1 \%$ (normal range $25-35 \%$ ). Iodide perchlorate discharge test indicated a block in organification $(43.5 \%)$ and the ${ }^{131}$ I scintiscan was compatible with Hashimoto's thyroiditis. Serum thyroid stimulating

Correspondence and present address: O. Modebe, B.Sc., M.D., Department of Medicine, University of Nigeria Teaching Hospital, P.M.B. 1129, Enugu, Nigeria

Accepted: 20 November 1985 hormone (TSH) concentration and T3 suppression test were normal. The serum antithyroglobulin titre was negative. She was treated with L-thyroxine for chronic thyroiditis associated with dysthyroid ophthalmopathy.

After 15 months, she developed signs and symptoms of thyrotoxicosis and investigations confirmed the presence of an overactive, autonomous thyroid gland. Thyroxine was discontinued and she was started on propylthiouracil and propanolol with clinical improvement. She refused ablative therapy and so was maintained on propylthiouracil for a possible remission.

Nine months later, she developed a cramping, periumbilical pain associated with fever and bloody diarrhoea. Propylthiouracil was discontinued. Investigations showed that she was anaemic but euthyroid. Sigmoidoscopy and barium enema were compatible with ulcerative colitis, and stool microscopy and culture revealed no pathogens. She responded to azulfidine (salicylazosulphapyridine) and prednisone.

Seven months later, she had an exacerbation of ulcerative colitis which was unresponsive to 2 weeks of treatment with large doses of prednisone and azulfidine. Thyroid function tests at that time revealed that she was thyrotoxic $(\mathrm{T} 4=13.0 \mu \mathrm{g} / \mathrm{dl}, \mathrm{T} 3=318 \mathrm{ng} / \mathrm{dl}$, T3 resin uptake $=39 \%$ ). Propylthiouracil and propranolol were added to her therapy and the abdominal symptoms improved within 2 weeks. She is now symptom-free on small doses of azulfidine, prednisone and propylthiouracil.

\section{Case 2}

A 38 year old Caucasian female complained of progressive weight loss, periorbital swelling, sleepless-

(C) The Fellowship of Postgraduate Medicine, 1986 
ness, anxiety and occasional vomiting for 4 months. On examination, she had mild periorbital oedema and chemosis. The thyroid gland was diffusely enlarged and firm.

Laboratory studies were normal except for a serum $\mathrm{T} 4 \mathrm{of} 15.0 \mu \mathrm{g} / \mathrm{dl}, \mathrm{T} 3 \mathrm{of} 328 \mathrm{ng} / \mathrm{dl}$ and resin T3 uptake of $44 \%$.

Treatment with propranolol and propylthiouracil produced steady improvement over the ensuing weeks. She elected to continue antithyroid therapy rather than undergo thyroid ablation. She was continued on $100 \mathrm{mg}$ of propylthiouracil daily for 12 months and felt well. The drug was then discontinued.

Approximately 5 months later, she became thyrotoxic again and was given $4.8 \mathrm{mCi}$ of ${ }^{131} \mathrm{I}$. Since then she has remained euthyroid.

Two years later, she developed abdominal pain, bloody diarrhoea and fever. Barium enema and sigmoidoscopy with biopsy confirmed the diagnosis of ulcerative colitis. Microscopic examination and culture of the stool revealed no infectious agents. Now, she is clinically well on azulfidine and prednisone and is euthyroid.

\section{Discussion}

The study by Janerot et al. (1975) is the most comprehensive report of the association of thyrotoxicosis and ulcerative colitis. Of 300 patients with ulcerative colitis, hyperthyroidism occurred in $3.8 \%$ compared to $0.8 \%$ of 600 control patients. In 7 of 11 patients who had both disorders, thyrotoxicosis preceded the ulcerative colitis, as in our cases but the reverse order does occur (Powell et al., 1968).

The factors responsible for this association are unknown but disordered autoimmunity has been postulated. Clubb et al. (1970) reported three young

\section{References}

BROWN, J. (Moderator) (1978). Autoimmune thyroid diseases - Graves' and Hashimoto's. Annals of Internal Medicine, 88, 379.

CLUBB, J.S., BLACK, P.J. \& WALlACE, D.C. (1970). An association of thyroid disease, ulcerative colitis and diabetes mellitus - a report of three cases in young women. Australian Annals of Medicine, 19, 159.

EDWARDS, F.C. \& TRUELOVE, S.C. (1964). The course and prognosis of ulcerative colitis - III. Complications. Gut, 5, 1.

JANEROT, G. (1975). The thyroid in ulcerative colitis and Crohn's disease - I Thyroid iodine uptake and urinary women with thyroid disease, diabetes mellitus and ulcerative colitis and suggested that a genetic or autoimmune factor was responsible for the association. All the cases so far reported have had Graves' disease, while one of our two patients also had Hashimoto's thyroiditis, both diseases being organspecific autoimmune disorders (Brown, 1978). There is also increasing evidence that an abnormal immune response may be responsible for some of the manifestations of ulcerative colitis (Jewell et al., 1972).

Abnormalities of iodine metabolism have been documented in patients with ulcerative colitis (Janerot, 1975). Although these are usually associated with a goitre they are unlikely to predispose to the development of Graves' disease.

There is no evidence that propylthiouracil plays any role in the development of colitis; some patients never received thiouracil drugs (Janerot et al., 1975), ulcerative colitis has preceded the development of Graves' disease, and in Case 1 of this report, propylthiouracil was used to control thyrotoxicosis with improvement in the colitis.

It has been emphasized that thyrotoxicosis makes the management of ulcerative colitis difficult as was exemplified in Case 1. The rapid metabolism of the drugs required to treat ulcerative colitis or the rapid transit of the drugs through the gut may prevent them attaining effective concentrations. Before ulcerative colitis in association with thyrotoxicosis can be managed, effective control of the thyrotoxicosis is essential. If control is not rapidly achieved, ablative therapy with radioactive iodine may be required. Some of the symptoms of acute ulcerative colitis may obscure the prompt diagnosis of concurrent thyrotoxicosis. If a patient with ulcerative colitis and a goitre is resistant to therapy, laboratory tests for thyrotoxicosis are indicated.

iodine excretion. Acta Medica Scandinavica, 197, 77.

JANEROT, G., AZAD KHAN, A.K. \& TRUELOVE, S.C. (1975). The thyroid in ulcerative colitis and Crohn's disease - II Thyroid enlargement and thyrotoxicosis in ulcerative colitis. Acta Medica Scandinavica, 197, 83.

JEWELL, D.P., MCLENNAN, I.C.M. \& TRUELOVE, S.C. (1972). Circulating immune complexes in ulcerative colitis and Crohn's disease. Gut, 13, 839.

POWELL, J.R., SHAPIRO, H.A. \& CARBONE, J.V. (1968). Therapeutic problems of ulcerative colitis with hyperthyroidism. American Journal of Gastroenterology, 50, 116. 